EUROPEAN JOURNAL OF PURE AND APPLIED MATHEMATICS

Vol. 13, No. 3, 2020, 645-662

ISSN 1307-5543 - www.ejpam.com

Published by New York Business Global

\title{
Some results on blow-up phenomenon for nonlinear porous medium equations with weighted source
}

\author{
Huafei $\mathrm{Di}^{1, *}$, Lin $\mathrm{Chen}^{2}$, Zefang Song ${ }^{3}$ \\ ${ }^{1}$ School of Mathematics and Information Science, Guangzhou University, Guangzhou 510006, \\ $P$ R China \\ ${ }^{2}$ College of Management and Economics, Tianjin University, Tianjin 300072, $P$ R China \\ ${ }^{3}$ School of Economics and Statistics, Guangzhou University, Guangzhou 510006, P R China
}

\begin{abstract}
This paper deals with the blow-up phenomena for a type of nonlinear porous medium equations with weighted source $u_{t}-\triangle u^{m}=a(x) f(u)$ subject to Dirichlet (or Neumann) boundary conditions. Based on the auxiliary functions and differential-integral inequalities, the blow-up criterions which ensure that $u$ cannot exist all time are given under two different assumptions, and the corresponding estimates on the upper bounds for blow-up time and blow-up rate are derived respectively. Moreover, we use three different methods to determine the lower bounds for blow-up time and blow-up rate estimates if blow-up does occurs.
\end{abstract}

2020 Mathematics Subject Classifications: 35A01, 35B44, 35K20, 35K61

Key Words and Phrases: Porous medium equation, Upper and lower bounds, Blow-up rate, Weighted source

\section{Introduction}

In this paper, we deal with the blow-up time and blow-up rate estimates of the solutions to the following problem:

$$
\begin{aligned}
& u_{t}-\triangle u^{m}=a(x) f(u), \quad x \in \Omega, \quad t>0, \\
& u(x, t)=0 \text { or } \frac{\partial u}{\partial \nu}=0, \quad x \in \partial \Omega, \quad t>0, \\
& u(x, 0)=g(x) \geq 0, \quad x \in \Omega,
\end{aligned}
$$

where $m>1$ and $\Omega \subset R^{n}(n \geq 3)$ is a smooth bounded domain, $\nu$ is the outward normal vector, $g(x)$ is a continuous nonnegative function and satisfies the compatible condition. Here, the nonlinear function $f$ satisfies

* Corresponding author.

DOI: https://doi.org/10.29020/nybg.ejpam.v13i3.3768

Email addresses: dihuafei@yeah.net (H.F. Di),

chenlinalbert@126.com (L. Chen), song-zefang@163.com (Z.F. Song). 
$\left(f_{1}\right): f(s) \geq 0$ for all $s \geq 0$;

And the weighted function $a(x) \in C^{1}(\Omega) \cap C^{0}(\bar{\Omega})$ satisfies

$\left(a_{1}\right): a(x) \geq C>0$ on $\bar{\Omega}$ for some constant $C$ or

$\left(a_{2}\right): a(x)>0$ in $\Omega$ and $a(x)=0$ on $\partial \Omega$.

It is well known that the porous medium equations have extensive physical background and rich theoretical connotation. They have been used to model the processes involving the chemical reaction, heat transfer or diffusion, population dynamics and so on. We refer readers to see $[1,26]$ and references therein, where a series of physical application of Eq.(1.1) are also summarized. For instance, the nonlinear term $f(u)$ of Eq.(1.1) describes the nonlinear source in the diffusion phenomena, and it is called to be "heat source". If the "heat source" occurs, the solutions of Eq.(1.1) might be unbounded at finite time, namely, the solutions might be blowing up in finite time.

The Eq.(1.1) includes many important physical models. If the exponent $m=1$ and weighted function $a(x) \equiv 1$, the model (1.1) reduces to the semilinear heat equations

$$
u_{t}-\triangle u=f(u), \quad x \in \Omega, \quad t>0 .
$$

About this model, many results about the blow-up phenomenon of the solutions have been obtained, we refer to see $[13,16-19,23,25]$ and references therein. In $[18,19]$, Payne and Schaefer obtained a lower bound on blow-up time of the solutions to the Eq.(1.4) under null Dirichlet boundary condition and homogeneous Neumann boundary condition, respectively. Later, Payne et al. [16, 17] studied the blow-up phenomenon of the solutions for Eq.(1.4) with nonlinear boundary conditions. When the nonlinear source term $f(u)=\int_{\Omega} u^{q} d x-k u^{s}$, Song [23] obtained the lower bounds for blow-up time of the solutions with either homogeneous Dirichlet or homogeneous Neumann boundary conditions in three dimensional space. Afterwards, Liu [13] studied the lower bounds for blow-up time under nonlinear boundary conditions in three dimensional space. In [25], Tang et al. extended the results of literature [13] in higher dimensional space.

When the exponent $m \neq 1$ and weighted function $a(x) \equiv 1$, the model (1.1) becomes the following porous medium equations

$$
u_{t}-\triangle u^{m}=f(u), \quad x \in \Omega, \quad t>0 .
$$

This type of equations appears in several branches of applied mathematics [10, 12]. There is large body of literature on the study of the Eq.(1.5), such as the existence and uniqueness in $[4,5,9,11,26]$, blow-up in $[5,7-10,26]$, asymptotic behavior in $[3,20,21,26]$ and other interesting results in $[2,4,12,26]$ and references therein. For instance, in the case of nonlinear source $f(u)=u^{p}$, Galaktionov et al. [5] obtained the finite time blow-up of the solutions for $1<p<m+\frac{2}{n}$, and proved the global existence of the solutions for $p>m+\frac{2}{n}$. For the critical case, Galaktionov and Levine [6], Kawanago [9] revealed that all nonnegative nontrivial mild solutions blow up in finite time. Jiang, Zheng and Song [8] gave some sufficient or necessary blow-up conditions, and the blow-up rate estimates, where $f(u)=u^{p}, m>1, p>1$. 
If the exponent $m=1$ and weighted function $a(x) \not \equiv 1$, the model (1.1) reduces to the semilinear parabolic equations with weighted source

$$
u_{t}-\triangle u=a(x) f(u), \quad x \in \Omega, \quad t>0 .
$$

Recently, the studying on the blow-up phenomenon had some new development, where more attention was paid on the parabolic equations with weighted source. These models can be used to illustrate the processes of heat transfer arising in physical and engineering applications, such as a model of phase separation in binary alloys [22]. The existence and nonexistence of global solutions, bounds for blow-up time, blow-up rate, blow-up sets and asymptotic behavior for this type of equations were investigated by many authors. We refer the reader to see $[14,15,24]$ and papers cited therein. For example, Song and Lv $[14,24]$ studied the initial boundary value problem for the above equations with nonlinear Neumann boundary condition, and they derived the upper and lower bounds for blow-up time in three dimensional space [14]. In [24], they further investigated the estimates of blow-up rate and the bounds for blow-up time in higher dimensional space. Ma and Fang [15] changed the diffusion term $\triangle u$ into $\sum_{i, j=1}^{N}\left(a^{i, j}(x) u_{x_{i}}\right)_{x_{j}}$ in Eq.(1.6), where the upper and lower bounds for the blow-up time were derived in higher dimensional space.

In the present work, we main study the blow-up phenomena for the porous medium equations with weighted nonlinear source. As far as we known, there is little information on the blow-up results of the solutions for problem (1.1)-(1.3). Obviously, the existence and uniqueness of local solutions for this problem can be obtained by applying the classical Faedo-Galerkin method or Contraction Mapping Principle. Naturally, we would like to study the estimates of blow-up rate and the bounds for blow-up time of the solutions in any smooth bounded domain $\Omega \subset R^{n}(n \geq 3)$. Here, the appearance of the diffusion term $\triangle u^{m}$ and weighted nonlinear source $a(x) f(u)$ cause some difficulties in dealing with the qualitative properties of problem (1.1)-(1.3). Hence, we shall use some modified auxiliary functions and differential-integral inequality skills to over these difficulties.

In detail, this paper is organized as follows: the blow-up criterions are given under two different assumptions, and the corresponding estimates on the upper bounds for blow-up time and blow-up rate are derived in Subsection 2.1 and 2.2. In section 3, we will use three methods to give the lower bounds for blow-up time and blow-up rate of the solutions if the blow-up does occurs.

\section{Upper estimates for blow-up time and blow-up rate}

The purpose of this section is to establish some estimates about the upper bounds for blow-up time and blow-up rate of the solutions to problem (1.1)-(1.3) under two different assumptions, respectively.

\subsection{The first method}

To obtain the results of this subsection, we first assume that 
$\left(f_{2}\right)$ : there exists a positive constant $C_{1}>2$ such that

$$
\int_{\Omega} a(x) s^{m} f(s) d x \geq C_{1} \int_{\Omega} a(x) F(s) d x
$$

for any function $s(x) \geq 0$, where $F(s)=m \int_{0}^{s} \theta^{m-1} f(\theta) d \theta$;

$\left(g_{1}\right)$ : the initial data $g(x)$ satisfies

$$
\int_{\Omega}\left|\nabla g^{m}\right|^{2} d x<2 \int_{\Omega} a(x) F(g) d x .
$$

Then, inspired by Payne et al. [16, 17], we further define the following auxiliary function

$$
\varphi(t)=\int_{\Omega} u^{m+1} d x
$$

Theorem 1. Assume that the conditions $\left(f_{1}\right),\left(f_{2}\right),\left(g_{1}\right),\left(a_{1}\right),\left(a_{2}\right)$ hold, and $u$ is a nonnegative solution of problem (1.1)-(1.3). Then, we conclude that the solution u becomes unbounded in $L^{m+1}$-norm at $t=t^{*}$. Moreover, an upper bound for blow-up time $t^{*}$ is given by

$$
t^{*} \leq \frac{(m+1) \varphi(0)}{(m-1) \phi(0)}
$$

and the upper estimate of blow-up rate can be given by

$$
\|u\|_{m+1} \leq\left(\frac{(m+1) \varphi(0)^{\frac{2 m}{m+1}}}{(m-1) \phi(0)}\right)^{\frac{1}{m-1}}\left(t^{*}-t\right)^{-\frac{1}{m-1}},
$$

where $\varphi(0)=\|g\|_{m+1}^{m+1}$ and $\phi(0)=-(m+1) \int_{\Omega}\left|\nabla g^{m}\right|^{2} d x+2(m+1) \int_{\Omega} a(x) F(g) d x>0$.

Proof. Firstly, differentiating (2.1) with respect to $t$ and using Eq.(1.1), then we have

$$
\begin{aligned}
\varphi^{\prime}(t) & =(m+1) \int_{\Omega} u^{m} u_{t} d x \\
& =(m+1) \int_{\Omega} u^{m}\left(\triangle u^{m}+a(x) f(u)\right) d x \\
& =-(m+1) \int_{\Omega}\left|\nabla u^{m}\right|^{2} d x+(m+1) \int_{\Omega} a(x) u^{m} f(u) d x .
\end{aligned}
$$

By the combination of (2.4) and condition $\left(f_{2}\right)$, we obtain

$$
\varphi^{\prime}(t) \geq-(m+1) \int_{\Omega}\left|\nabla u^{m}\right|^{2} d x+C_{1}(m+1) \int_{\Omega} a(x) F(u) d x>\phi(t),
$$

where

$$
\phi(t)=-(m+1) \int_{\Omega}\left|\nabla u^{m}\right|^{2} d x+2(m+1) \int_{\Omega} a(x) F(u) d x .
$$


On the other hand, a simple computation yields

$$
\begin{aligned}
\phi^{\prime}(t)= & -2(m+1) \int_{\Omega} \nabla u^{m} \cdot\left(\nabla u^{m}\right)_{t} d x \\
& +2 m(m+1) \int_{\Omega} a(x) u^{m-1} u_{t} f(u) d x \\
= & 2 m(m+1) \int_{\Omega} u^{m-1} u_{t}\left(\triangle u^{m}+a(x) f(u)\right) d x \\
= & 2 m(m+1) \int_{\Omega} u^{m-1} u_{t}^{2} d x \geq 0 .
\end{aligned}
$$

Here, we have used the fact that $u(x, t)=0$ (or $\frac{\partial u}{\partial \nu}=0$ ) on $\partial \Omega$. Using Schwarz's inequality, we get

$$
\left(\int_{\Omega} u^{m} u_{t} d x\right)^{2} \leq \int_{\Omega} u^{m+1} d x \int_{\Omega} u^{m-1} u_{t}^{2} d x
$$

Hence, multiplying $\varphi(t)$ by $\phi^{\prime}(t)$, it follows from (2.5) that

$$
\begin{aligned}
\varphi(t) \phi^{\prime}(t) & =2 m(m+1) \int_{\Omega} u^{m+1} d x \int_{\Omega} u^{m-1} u_{t}^{2} d x \\
& \geq 2 m(m+1)\left(\int_{\Omega} u^{m} u_{t} d x\right)^{2}=\frac{2 m}{m+1}\left[\varphi^{\prime}(t)\right]^{2} \\
& \geq \frac{2 m}{m+1} \varphi^{\prime}(t) \phi(t) .
\end{aligned}
$$

Thus, the above inequality implies that

$$
\left(\phi(t)[\varphi(t)]^{-\frac{2 m}{m+1}}\right)^{\prime}=[\varphi(t)]^{-\frac{3 m+1}{m+1}}\left\{\varphi(t) \phi^{\prime}(t)-\frac{2 m}{m+1} \varphi^{\prime}(t) \phi(t)\right\} \geq 0 .
$$

Utilizing the assumption $\left(g_{1}\right)$ and $(2.1),(2.7)$, we know that

$$
\varphi(0)=\|g\|_{m+1}^{m+1}>0
$$

and

$$
\phi(t) \geq \phi(0)=-(m+1) \int_{\Omega}\left|\nabla g^{m}\right|^{2} d x+2(m+1) \int_{\Omega} a(x) F(g) d x>0 .
$$

Integrating (2.10) from 0 to $t$, we obtain

$$
\phi(t)[\varphi(t)]^{-\frac{2 m}{m+1}} \geq \phi(0)[\varphi(0)]^{-\frac{2 m}{m+1}}=M>0 .
$$

By (2.5) and (2.13), we get

$$
\frac{m+1}{1-m}\left([\varphi(t)]^{\frac{1-m}{m+1}}\right)^{\prime}=\varphi^{\prime}(t)[\varphi(t)]^{-\frac{2 m}{m+1}} \geq \phi(t)[\varphi(t)]^{-\frac{2 m}{m+1}}>0 .
$$


Integrating (2.14) from 0 to $t$, we have

$$
[\varphi(t)]^{\frac{1-m}{m+1}} \leq[\varphi(0)]^{\frac{1-m}{m+1}}-\frac{m-1}{m+1} M t .
$$

Clearly, the above inequality cannot hold for all $t>0$. Consequently, $u$ blows up at some finite time $t^{*}$ and

$$
t^{*} \leq \frac{m+1 \varphi(0)}{(m-1) \phi(0)}
$$

Furthermore, from (2.5) and (2.13) again, we have

$$
\varphi^{\prime}(t) \geq \phi(t) \geq \phi(0)[\varphi(0)]^{-\frac{2 m}{m+1}}[\varphi(t)]^{\frac{2 m}{m+1}} .
$$

Integrating (2.16) from $t$ to $t^{*}$, we obtain

$$
\varphi(t) \leq\left(\frac{(m+1) \varphi(0)^{\frac{2 m}{m+1}}}{(m-1) \phi(0)}\right)^{\frac{m+1}{m-1}}\left(t^{*}-t\right)^{-\frac{m+1}{m-1}}
$$

which implies that the upper estimate of blow-up rate is given by $(2.3)$.

\subsection{The second method}

We first assume that

$\left(f_{3}\right)$ : there exists a positive function $G(\theta)$ such that

$$
\int_{\Omega} a(x) f(s) d x \geq C_{2} G\left[\int_{\Omega} s d x\right] \text { with } \int_{0}^{+\infty} \frac{d \theta}{G(\theta)}<+\infty,
$$

for any function $s(x) \geq 0$. Then, we define the following auxiliary function

$$
\varphi_{1}(t)=\int_{\Omega} u d x
$$

Theorem 2. Assume that the conditions $\left(f_{1}\right),\left(f_{3}\right),\left(a_{1}\right),\left(a_{2}\right)$ hold, and $u$ is a nonnegative solution of problem (1.1)-(1.3). Then, we conclude that the solution u becomes unbounded in $L^{1}$-norm at $t=t^{*}$. Moreover, an upper bound for blow-up time $t^{*}$ is given by

$$
t^{*} \leq \int_{\varphi_{1}(0)}^{+\infty} \frac{d \theta}{G(\theta)}<+\infty
$$

and the upper estimate of blow-up rate can be given by

$$
\|u\|_{L^{1}} \leq Y^{-1}\left(t^{*}-t\right)
$$

where the function $Y(s):=\int_{s}^{+\infty} \frac{d \theta}{G(\theta)}$ for any function $s(x) \geq 0$, and $\varphi_{1}(0)=\int_{\Omega} g d x$. 
Proof. Integrating the Eq.(1.1) by parts, from the condition $\left(f_{3}\right)$ and $(2.18)$ we have

$$
\int_{\Omega} u_{t} d x=\int_{\Omega} a(x) f(u) d x \geq C_{2} G\left[\int_{\Omega} u d x\right]
$$

which means that

$$
\varphi_{1}^{\prime}(t) \geq C_{2} G\left[\varphi_{1}(t)\right]>0
$$

Here, we have used the fact that $u(x, t)=0$ ( or $\left.\frac{\partial u}{\partial \nu}=0\right)$ on $\partial \Omega$. It then follows from (2.22) that $\varphi_{1}(t)$ is a increasing function, so we have

$$
\varphi_{1}(t)>\varphi_{1}(0)=\int_{\Omega} g(x) d x \geq 0 .
$$

Integrating (2.22) from 0 to $\mathrm{t}$ and using $(2.23),\left(f_{3}\right)$, we discover

$$
t \leq \int_{\varphi_{1}(0)}^{\varphi_{1}(t)} \frac{d \theta}{G(\theta)} \leq \int_{\varphi_{1}(0)}^{+\infty} \frac{d \theta}{G(\theta)}<+\infty .
$$

Obviously, (2.24) cannot hold for all time $t$. Consequently, we can derive an upper bound $t^{*}$ such that

$$
t^{*} \leq \int_{\varphi_{1}(0)}^{+\infty} \frac{d \theta}{G(\theta)}<+\infty
$$

and

$$
\lim _{t \rightarrow t^{*}} \varphi_{1}(t)=+\infty
$$

where $\left(0, t^{*}\right)$ is the interval of existence of the solutions $u$ in $L^{1}$-norm. In fact, if the equality (2.25) doesn't hold, then there exists a time $t_{1}>t^{*}$ such that $\varphi_{1}\left(t^{*}\right)<\varphi_{1}\left(t_{1}\right)<$ $+\infty$ and $t_{1}$ satisfies the inequality (2.23),(2.24), which contradict the maximum existence of $t^{*}$.

Furthermore, integrating (2.22) from $\mathrm{t}$ to $t^{*}$, it follows that

$$
t^{*}-t \leq \int_{\varphi_{1}(t)}^{+\infty} \frac{d \theta}{G(\theta)}:=Y\left(\varphi_{1}(t)\right)
$$

We note that $Y$ is a decreasing function, which means its inverse function $Y^{-1}$ exists and is also a decreasing function. Therefore, we have

$$
\varphi_{1}(t) \leq Y^{-1}\left(t^{*}-t\right)
$$

which implies that the estimate (2.20) of blow-up rate holds.

Remark 1. This result can be generalized to the case of problem (1.1)-(1.3) subject to $\frac{\partial u}{\partial \nu}=b(x, t) \geq 0$. In this case, it follows that

$$
\varphi_{1}^{\prime}(t) \geq m \int_{\partial \Omega} u^{m-1} b(x, t) d s+C_{2} G\left[\varphi_{1}(t)\right]>0 .
$$

We also can obtain the inequalities (2.19) and (2.20). 


\section{Lower estimates for blow-up time and blow-up rate}

In this section, we will give three methods to establish the lower bounds for blow-up time and blow-up rate of the solution to problem (1.1)-(1.3).

\subsection{The first method}

Firstly, let us assume that

$\left(f_{4}\right)$ : there exists positive constants $C_{3}, C_{4}$ such that

$$
a(x) f(s) \leq C_{3}+C_{4} s^{l+1},
$$

for any function $s(x) \geq 0$, where $0<l \leq \frac{2 n m-(n-2)(m+1)}{n}$. And then we introduce the auxiliary function $\varphi(t)=\int_{\Omega} u^{m+1} d x$ as $(2.1)$.

Next, we shall state and prove the main results of this subsection as follows:

Theorem 3. Assume that the conditions $\left(f_{1}\right),\left(f_{4}\right),\left(a_{1}\right),\left(a_{2}\right)$ hold, and $u$ is a nonnegative solution of problem (1.1)-(1.3) which becomes unbounded in $L^{m+1}-n o r m$ at $t=t^{*}$. Then, we conclude that a lower bound for blow-up time $t^{*}$ is given by

$$
t^{*} \geq \int_{\varphi(0)}^{+\infty} \frac{d \eta}{k_{1} \eta+k_{2} \eta^{1+\frac{2}{n \epsilon_{2}}}} .
$$

and the lower estimate of blow-up rate is

$$
\|u\|_{m+1} \geq\left(\frac{4 k_{2}}{n \epsilon_{2}}\right)^{-\frac{n \epsilon_{2}}{2(m+1)}}\left(t^{*}-t\right)^{-\frac{n \epsilon_{2}}{2(m+1)}},
$$

where $k_{1}, k_{2}$ and $\epsilon_{2}$ are positive constants which will be given later.

Proof. Differentiating (2.1) with respect to $t$ and using the condition $\left(f_{4}\right)$ and (1.1), we have

$$
\begin{aligned}
\varphi^{\prime}(t)= & (m+1) \int_{\Omega} u^{m} u_{t} d x \\
= & (m+1) \int_{\Omega} u^{m}\left(\triangle u^{m}+a(x) f(u)\right) d x \\
= & -(m+1) \int_{\Omega}\left|\nabla u^{m}\right|^{2} d x+(m+1) \int_{\Omega} a(x) u^{m} f(u) d x \\
\leq & -(m+1) \int_{\Omega}\left|\nabla u^{m}\right|^{2} d x+(m+1) C_{3} \int_{\Omega} u^{m} d x \\
& +(m+1) C_{4} \int_{\Omega} u^{m+l+1} d x .
\end{aligned}
$$

From Hölder's inequality, Young's inequality and condition $\left(f_{4}\right)$, we have

$$
\int_{\Omega} u^{m} d x \leq \frac{m}{m+l+1} \int_{\Omega} u^{m+l+1} d x+\frac{l+1}{m+l+1}|\Omega|,
$$


and

$$
\begin{aligned}
\int_{\Omega} u^{m+l+1} d x= & \int_{\Omega} u^{m+1-\epsilon_{1}} u^{l-\epsilon_{1}} d x \\
\leq & \left(\int_{\Omega} u^{m+1} d x\right)^{\frac{m+1-\epsilon_{1}}{m+1}}\left(\int_{\Omega} u^{\frac{\left(l+\epsilon_{1}\right)(m+1)}{\epsilon_{1}}} d x\right)^{\frac{\epsilon_{1}}{m+1}} \\
= & \left(\int_{\Omega} u^{m+1} d x\right)^{\frac{m+1-\epsilon_{1}}{m+1}}\left(\int_{\Omega} u^{\frac{2 n m}{n-2}} d x\right)^{\frac{\epsilon_{1}}{m+1}} \\
\leq & \frac{\epsilon_{2} C(\epsilon)}{1+\epsilon_{2}}\left(\int_{\Omega} u^{m+1} d x\right)^{\frac{\left(m+1-\epsilon_{1}\right)\left(\epsilon_{2}+1\right)}{(m+1) \epsilon_{2}}} \\
& +\frac{\epsilon}{1+\epsilon_{2}}\left(\int_{\Omega} u^{\frac{2 n m}{n-2}} d x\right)^{\frac{\epsilon_{1}\left(1+\epsilon_{2}\right)}{m+1}},
\end{aligned}
$$

where $\epsilon_{1}=\frac{(n-2)(m+1) l}{2 n m-(n-2)(m+1)}>0, \epsilon_{2}=\frac{n(2 m-l)-(n-2)(m+1)}{n l}>0$, and $\epsilon$ will be determined later.

Noting that

$$
\int_{\Omega} u^{\frac{2 n m}{n-2}} d x \leq C_{5}^{\frac{2 n}{n-2}}\left(\int_{\Omega}\left|\nabla u^{m}\right|^{2}\right)^{\frac{n}{n-2}}
$$

where $C_{5}$ is the optimal constant of the Sobolev embedding $H^{1}(\Omega) \hookrightarrow L^{\frac{2 n}{n-2}}(\Omega)$.

Furthermore, from the choice of $\epsilon_{1}$ and $\epsilon_{2}$, it is easy to see that $\frac{\epsilon_{1}\left(1+\epsilon_{2}\right) n}{(m+1)(n-2)}=1$. Inserting (3.4)-(3.6) into (3.3), it follows that

$$
\begin{aligned}
\varphi^{\prime}(t) \leq & -(m+1) \int_{\Omega}\left|\nabla u^{m}\right|^{2} d x+\frac{(m+1) C_{3}(l+1)}{m+l+1}|\Omega| \\
& +\left[\frac{m(m+1) C_{3}}{m+l+1}+(m+1) C_{4}\right] \int_{\Omega} u^{m+l+1} d x \\
\leq & -\left[m+1-\frac{(m+1)\left(m C_{3}+m C_{4}+l C_{4}+C_{4}\right) C_{5}^{2} \epsilon}{\left(1+\epsilon_{2}\right)(m+l+1)}\right] \int_{\Omega}\left|\nabla u^{m}\right|^{2} d x \\
& +\frac{(m+1)\left(m C_{3}+m C_{4}+l C_{4}+C_{4}\right) \epsilon_{2} C(\epsilon)}{\left(1+\epsilon_{2}\right)(m+l+1)}\left(\int_{\Omega} u^{m+1} d x\right)^{\frac{\left(m+1-\epsilon_{1}\right)\left(\epsilon_{2}+1\right)}{(m+1) \epsilon_{2}}} \\
& +\frac{(m+1) C_{3}(l+1)}{m+l+1}|\Omega| .
\end{aligned}
$$

Taking $\epsilon$ small enough such that

$$
m+1-\frac{(m+1)\left(m C_{3}+m C_{4}+l C_{4}+C_{4}\right) C_{5}^{2} \epsilon}{\left(1+\epsilon_{2}\right)(m+l+1)}>0 .
$$

Hence, we have

$$
\varphi^{\prime}(t) \leq k_{1}+k_{2}[\varphi(t)]^{\frac{\left(m+1-\epsilon_{1}\right)\left(1+\epsilon_{2}\right)}{(m+1) \epsilon_{2}}}=k_{1}+k_{2}[\varphi(t)]^{1+\frac{2}{n \epsilon_{2}}},
$$

where $k_{1}=\frac{(m+1) C_{3}(l+1)}{m+l+1}|\Omega|$ and $k_{2}=\frac{(m+1)\left(m C_{3}+m C_{4}+l C_{4}+C_{4}\right) \epsilon_{2} C(\epsilon)}{\left(1+\epsilon_{2}\right)(m+l+1)}$. 
Integrating (3.9) from 0 to t, we get

$$
\int_{\varphi(0)}^{\varphi(t)} \frac{d \eta}{k_{1} \eta+k_{2} \eta^{1+\frac{2}{n \epsilon_{2}}}} \leq t .
$$

If $u$ blows up in the measure $\varphi(t)$ as $t \rightarrow t^{*}$, then we can obtain the lower bound

$$
t^{*} \geq \int_{\varphi(0)}^{+\infty} \frac{d \eta}{k_{1} \eta+k_{2} \eta^{1+\frac{2}{n \epsilon_{2}}}} .
$$

Moreover, integrating the inequality (3.9) from t to $t^{*}$, we obtain

$$
t^{*}-t \geq \int_{\varphi(t)}^{+\infty} \frac{d \eta}{k_{1} \eta+k_{2} \eta^{1+\frac{2}{n \epsilon_{2}}}}:=Y_{1}(\varphi(t)) .
$$

We note that $Y_{1}$ is a decreasing function, which means its inverse function $Y_{1}^{-1}$ exists and is also a decreasing function. Therefore, we have

$$
\varphi(t) \geq Y_{1}^{-1}\left(t^{*}-t\right)
$$

which gives the lower estimate of blow-up rate. In fact, if $t$ closes $t^{*}$ enough such that $\varphi(t) \gg 1$ and $k_{2} \eta^{1+\frac{2}{n \epsilon_{2}}}>k_{1} \eta$ in the inequality (3.11), then we have

$$
t^{*}-t \geq \frac{n \epsilon_{2}}{4 k_{2}}[\varphi(t)]^{-\frac{2}{n \epsilon_{2}}}
$$

which means that

$$
\varphi(t) \geq\left(\frac{4 k_{2}}{n \epsilon_{2}}\right)^{-\frac{n \epsilon_{2}}{2}}\left(t^{*}-t\right)^{-\frac{n \epsilon_{2}}{2}} .
$$

Thus, the estimate (3.2) of blow-up rate also holds.

\subsection{The second method}

Firstly, we need the following assumption:

$\left(f_{5}\right)$ : there exists positive constants $C_{6}, C_{7}, q$ and $Q$ such that

$$
a(x) f(s) \leq C_{6}+C_{7} s^{p}\left(\int_{\Omega} s^{q+1} d x\right)^{Q}
$$

for any function $s(x) \geq 0$.

$\left(e_{1}\right)$ : we also assume that

$$
0 \leq p \leq 1, \quad 0 \leq q \leq m, \text { and }(q+1) Q+p>1 .
$$

To obtain the main results, we define the auxiliary function $\varphi(t)=\int_{\Omega} u^{m+1} d x$ again.

Next, we will state our results below: 
Theorem 4. Assume that the conditions $\left(f_{1}\right),\left(f_{5}\right),\left(e_{1}\right),\left(a_{1}\right),\left(a_{2}\right)$ hold, and $u$ is a nonnegative solution of problem (1.1)-(1.3) which becomes unbounded in $L^{m+1}$-norm at $t=t^{*}$. Then, we conclude that a lower bound for blow-up time $t^{*}$ is given by

$$
t^{*} \geq \int_{\varphi(0)}^{+\infty} \frac{d \eta}{k_{3} \eta^{\frac{m}{m+1}}+k_{4} \eta^{\frac{m+p+(q+1) Q}{m+1}}},
$$

and the lower estimate of blow-up rate is

$$
\|u\|_{m+1} \geq\left[\frac{2 k_{4}((q+1) Q+p-1)}{m+1}\right]^{-\frac{1}{(q+1) Q+p-1}}\left(t^{*}-t\right)^{-\frac{1}{(q+1) Q+p-1}},
$$

where $k_{3}=C_{6}(m+1)|\Omega|^{\frac{1}{m+1}}, k_{4}=C_{7}(m+1)|\Omega|^{\frac{1-p+(m-q) Q}{m+1}}$.

Proof. Under the assumption condition $\left(f_{5}\right)$, we have from (1.1) and (2.1) that

$$
\begin{aligned}
\varphi^{\prime}(t) & =(m+1) \int_{\Omega} u^{m} u_{t} d x \\
& =(m+1) \int_{\Omega} u^{m}\left(\triangle u^{m}+a(x) f(u)\right) d x \\
& =-(m+1) \int_{\Omega}\left|\nabla u^{m}\right|^{2} d x+(m+1) \int_{\Omega} a(x) u^{m} f(u) d x \\
& \leq C_{6}(m+1) \int_{\Omega} u^{m} d x+C_{7}(m+1) \int_{\Omega} u^{m+p} d x\left(\int_{\Omega} u^{q+1} d x\right)^{Q} .
\end{aligned}
$$

Applying Hölder's inequality and condition $\left(e_{1}\right)$, we know that

$$
\begin{gathered}
\int_{\Omega} u^{m} d x \leq\left(\int_{\Omega} u^{m+1} d x\right)^{\frac{m}{m+1}}|\Omega|^{\frac{1}{m+1}} \\
\int_{\Omega} u^{m+p} d x \leq\left(\int_{\Omega} u^{m+1} d x\right)^{\frac{m+p}{m+1}}|\Omega|^{\frac{1-p}{m+1}}
\end{gathered}
$$

and

$$
\int_{\Omega} u^{q+1} d x \leq\left(\int_{\Omega} u^{m+1} d x\right)^{\frac{q+1}{m+1}}|\Omega|^{\frac{m-q}{m+1}} .
$$

Inserting (3.18)-(3.20) into (3.17), it follows that

$$
\begin{aligned}
\varphi^{\prime}(t) \leq & C_{6}(m+1)|\Omega|^{\frac{1}{m+1}}\left(\int_{\Omega} u^{m+1} d x\right)^{\frac{m}{m+1}} \\
& +C_{7}(m+1)|\Omega|^{\frac{1-p+(m-q) Q}{m+1}}\left(\int_{\Omega} u^{m+1} d x\right)^{\frac{m+p+(q+1) Q}{m+1}},
\end{aligned}
$$


where from the condition $\left(e_{1}\right)$, it is easy to see that $\frac{m+p+(q+1) Q}{m+1}>1$.

Then, integrating the above inequality from 0 to $t$ yields that

$$
\int_{\varphi(0)}^{\varphi(t)} \frac{d \eta}{k_{3} \eta^{\frac{m}{m+1}}+k_{4} \eta^{\frac{m+p+(q+1) Q}{m+1}}} \leq t,
$$

where $k_{3}=C_{6}(m+1)|\Omega|^{\frac{1}{m+1}}, k_{4}=C_{7}(m+1)|\Omega|^{\frac{1-p+(m-q) Q}{m+1}}$.

If $u$ blows up in the measure $\varphi(t)$ as $t \rightarrow t^{*}$, then we can obtain the lower bound

$$
t^{*} \geq \int_{\varphi(0)}^{+\infty} \frac{d \eta}{k_{3} \eta^{\frac{m}{m+1}}+k_{4} \eta^{\frac{m+p+(q+1) Q}{m+1}}} .
$$

Furthermore, integrating the inequality (3.21) from $t$ to $t^{*}$, we obtain

$$
t^{*}-t \geq \int_{\varphi(t)}^{+\infty} \frac{d \eta}{k_{3} \eta^{\frac{m}{m+1}}+k_{4} \eta^{\frac{m+p+(q+1) Q}{m+1}}}:=Y_{2}(\varphi(t)) .
$$

We note that $Y_{2}$ is a decreasing function, which means its inverse function $Y_{2}^{-1}$ exists and it is also a decreasing function. Therefore, we have

$$
\varphi(t) \geq Y_{2}^{-1}\left(t^{*}-t\right)
$$

which gives the lower estimate of blow-up rate. In fact, the auxiliary function $\varphi(t)$ becomes unbounded at time $t=t^{*}$, so we know that $\varphi(t) \gg 1$ and the inequality $k_{4} \eta^{\frac{m+p+(q+1) Q}{m+1}}>$ $k_{3} \eta^{\frac{m}{m+1}}$ as $t \rightarrow t^{*-}$. Hence, when $t$ is close to $t^{*}$, inserting the above inequality into (3.22) and then a direct calculation yields that

$$
t^{*}-t \geq \frac{m+1}{2 k_{4}[(q+1) Q+p-1]}[\varphi(t)]^{-\frac{(q+1) Q+p-1}{m+1}},
$$

which means that

$$
\varphi(t) \geq\left[\frac{2 k_{4}((q+1) Q+p-1)}{m+1}\right]^{-\frac{m+1}{(q+1) Q+p-1}}\left(t^{*}-t\right)^{-\frac{m+1}{(q+1) Q+p-1}} .
$$

Hence, the estimate (3.16) of blow-up rate holds.

\subsection{The third method}

This subsection is devoted to the estimates of the lower bounds for blow-up time and blow-up rate of the solutions to problem (1.1)-(1.3) by utilizing the method appearing in $[18,19]$.

For this purpose, we need to assume that

$\left(f_{6}\right): s^{m-1} \geq \alpha\left(\int_{s}^{+\infty} \frac{d \eta}{f(\eta)}\right)^{-\gamma}$, for any function $s(x) \geq 0$, where $\alpha, \gamma$ are positive constants and $0<\gamma<1$; 
$\left(f_{7}\right)$ : there exist positive constants $k$ and $\beta$ such that $k>\frac{4(n-2)-n \gamma}{2 n}$,

$$
f(s)\left(\int_{s}^{+\infty} \frac{d \eta}{f(\eta)}\right)^{n k+1} \rightarrow+\infty, \quad \text { as } s \rightarrow 0^{+},
$$

and

$$
f^{\prime}(s) \int_{s}^{+\infty} \frac{d \eta}{f(\eta)} \leq n k+1-\beta
$$

for any function $s(x) \geq 0$. Then, we define the following auxiliary function

$$
\varphi_{2}(t)=\int_{\Omega} V^{n k}(u) d x, \quad V(u)=\left(\int_{u}^{+\infty} \frac{d \eta}{f(\eta)}\right)^{-1} .
$$

Next, we will state our results below:

Theorem 5. Assume that the conditions $\left(f_{1}\right),\left(f_{6}\right),\left(f_{7}\right),\left(a_{1}\right),\left(a_{2}\right)$ hold, and $u$ is a nonnegative solution of problem (1.1)-(1.3) which becomes unbounded in $\varphi_{2}(t)$-form at $t=t^{*}$. Then, we conclude that a lower bound for blow-up time $t^{*}$ is given by

$$
t^{*} \geq \int_{\varphi_{2}(0)}^{+\infty} \frac{d \eta}{k_{5}+k_{6} \eta^{\frac{3 n-6}{3 n-8}}},
$$

and the lower estimate of blow-up rate can be given by

$$
\varphi_{2}(t) \geq\left(\frac{4 k_{6}}{3 n-8}\right)^{-\frac{3 n-8}{2}}\left(t^{*}-t\right)^{-\frac{3 n-8}{2}},
$$

where $k_{5}, k_{6}$ will be given later, and $\varphi_{2}(0)=\int_{\Omega}\left[\int_{g}^{+\infty} \frac{d \eta}{f(\eta)}\right]^{-n k} d x$.

Proof. Under the assumptions $\left(f_{7}\right)$, we have from (1.1) and (3.26) that

$$
\begin{aligned}
\varphi_{2}^{\prime}(t)= & n k \int_{\Omega} V^{n k+1}[f(u)]^{-1} u_{t} d x \\
= & n k \int_{\Omega} V^{n k+1}[f(u)]^{-1}\left[\triangle u^{m}+a(x) f(u)\right] d x \\
= & -m n k(n k+1) \int_{\Omega} V^{n k+2}[f(u)]^{-2}|\nabla u|^{2} u^{m-1} d x+n k \int_{\Omega} V^{n k+1} a(x) d x \\
& +m n k \int_{\Omega} V^{n k+1}[f(u)]^{-2} f^{\prime}(u) u^{m-1}|\nabla u|^{2} d x . \\
\leq & -m n k(n k+1) \int_{\Omega} V^{n k+2}[f(u)]^{-2}|\nabla u|^{2} u^{m-1} d x+n k \int_{\Omega} V^{n k+1} a(x) d x \\
& +m n k(n k+1-\beta) \int_{\Omega} V^{n k+2}[f(u)]^{-2} u^{m-1}|\nabla u|^{2} d x
\end{aligned}
$$


H.F. Di, L. Chen and Z.F. Song / Eur. J. Pure Appl. Math, 13 (3) (2020), 645-662

$$
=n k \int_{\Omega} V^{n k+1} a(x) d x-m n k \beta \int_{\Omega} V^{n k+2}[f(u)]^{-2} u^{m-1}|\nabla u|^{2} d x .
$$

Since

$$
\left|\nabla V^{\frac{n k+\gamma}{2}}\right|^{2}=\left(\frac{n k+\gamma}{2}\right)^{2} V^{n k+\gamma+2}[f(u)]^{-2}|\nabla u|^{2}
$$

In view of assumptions $\left(f_{6}\right),(3.29)$ and $(3.30)$, we discover that

$$
\varphi_{2}^{\prime}(t) \leq n k \int_{\Omega} V^{n k+1} a(x) d x-\frac{4 n k \alpha \beta}{(n k+\gamma)^{2}} \int_{\Omega}\left|\nabla V^{\frac{n k+\gamma}{2}}\right|^{2} d x .
$$

For convenience, we denote

$$
\begin{aligned}
\epsilon_{3} & =\frac{3 n k(n-2)+n(n k+\gamma)-4(n-2)(n k+1)}{3 n k(n-2)+n(n k+\gamma)}, \\
\epsilon_{4} & =\frac{4(n-2)(n k+1)}{3 n k(n-2)+n(n k+\gamma)},
\end{aligned}
$$

where the assumptions $\left(f_{7}\right)$ implies that $\epsilon_{3}>0, \epsilon_{4}>0$ and $\epsilon_{3}+\epsilon_{4}=1$. So by Hölder's inequality, we have

$$
\int_{\Omega} V^{n k+1} a(x) d x \leq\left(\int_{\Omega} V^{\frac{3 n k(n-2)+n(n k+\gamma)}{4(n-2)}} d x\right)^{\epsilon_{4}}\left(\int_{\Omega} a(x)^{\frac{1}{\epsilon_{3}}} d x\right)^{\epsilon_{3}} .
$$

Using Hölder's inequality again, it follows that

$$
\int_{\Omega} V^{\frac{3 n k(n-2)+n(n k+\gamma)}{4(n-2)}} d x \leq\left(\int_{\Omega} V^{n k} d x\right)^{\frac{3}{4}}\left(\int_{\Omega} V^{\frac{n(n k+\gamma)}{n-2}} d x\right)^{\frac{1}{4}} .
$$

Furthermore, applying the Sobolev's inequality, we obtain that

$$
\left(\int_{\Omega} V^{\frac{n(n k+\gamma)}{n-2}} d x\right)^{\frac{n-2}{2 n}}=\left(\int_{\Omega}\left(V^{\frac{n k+\gamma}{2}}\right)^{\frac{2 n}{n-2}} d x\right)^{\frac{n-2}{2 n}} \leq C_{5}\left(\int_{\Omega}\left|\nabla V^{\frac{n k+\gamma}{2}}\right|^{2} d x\right)^{\frac{1}{2}}
$$

where $C_{5}$ is the optimal Sobolev's embedding constant defined as (3.6). Substituting (3.34) into (3.33) yields that

$$
\int_{\Omega} V^{\frac{3 n k(n-2)+n(n k+\gamma)}{4(n-2)}} d x \leq C_{5}^{\frac{n}{2(n-2)}}\left(\int_{\Omega} V^{n k} d x\right)^{\frac{3}{4}}\left(\int_{\Omega}\left|\nabla V^{\frac{n k+\gamma}{2}}\right|^{2} d x\right)^{\frac{n}{4(n-2)}} .
$$

By Young's inequality, (3.32) and (3.35), we obtain

$$
\int_{\Omega} V^{n k+1} a(x) d x
$$


H.F. Di, L. Chen and Z.F. Song / Eur. J. Pure Appl. Math, 13 (3) (2020), 645-662

$$
\begin{aligned}
& \leq\left(C_{5}^{\frac{n}{2(n-2)}}\left(\int_{\Omega} V^{n k} d x\right)^{\frac{3}{4}}\left(\int_{\Omega}\left|\nabla V^{\frac{n k+\gamma}{2}}\right|^{2} d x\right)^{\frac{n}{4(n-2)}}\right)^{\epsilon_{4}}\left(\int_{\Omega} a(x)^{\frac{1}{\epsilon_{3}}} d x\right)^{\epsilon_{3}} \\
& \leq \epsilon_{4} C_{5}^{\frac{n}{2(n-2)}}\left(\int_{\Omega} V^{n k} d x\right)^{\frac{3}{4}}\left(\int_{\Omega}\left|\nabla V^{\frac{n k+\gamma}{2}}\right|^{2} d x\right)^{\frac{n}{4(n-2)}}+\epsilon_{3} \int_{\Omega} a(x)^{\frac{1}{\epsilon_{3}}} d x .
\end{aligned}
$$

Applying Young's inequality again, we have

$$
\begin{aligned}
& \left(\int_{\Omega} V^{n k} d x\right)^{\frac{3}{4}}\left(\int_{\Omega}\left|\nabla V^{\frac{n k+\gamma}{2}}\right|^{2} d x\right)^{\frac{n}{4(n-2)}} \\
& \leq \frac{n \delta}{4(n-2)} \int_{\Omega}\left|\nabla V^{\frac{n k+\gamma}{2}}\right|^{2} d x+\frac{(3 n-8) C(\delta)}{4(n-2)}\left(\int_{\Omega} V^{n k} d x\right)^{\frac{3 n-6}{3 n-8}},
\end{aligned}
$$

where $\frac{n}{4(n-2)}>1, \frac{3 n-8}{4(n-2)}>1$ with $\frac{n}{4(n-2)}+\frac{3 n-8}{4(n-2)}=1$.

Consequently, it follows that

$$
\begin{aligned}
\int_{\Omega} V^{n k+1} a(x) d x \leq & \epsilon_{3} \int_{\Omega} a(x)^{\frac{1}{\epsilon_{3}}} d x+\frac{\epsilon_{4} n \delta}{4(n-2)} \int_{\Omega}\left|\nabla V^{\frac{n k+\gamma}{2}}\right|^{2} d x \\
& +\frac{\epsilon_{4}(3 n-8) C(\delta)}{4(n-2)}\left(\int_{\Omega} V^{n k} d x\right)^{\frac{3 n-6}{3 n-8}} .
\end{aligned}
$$

Substituting (3.38) into (3.29), we get

$$
\begin{aligned}
\varphi_{2}^{\prime}(t) \leq & \epsilon_{3} \int_{\Omega} a(x)^{\frac{1}{\epsilon_{3}}} d x+\frac{\epsilon_{4} n k(3 n-8) C(\delta)}{4(n-2)}\left(\int_{\Omega} V^{n k} d x\right)^{\frac{3 n-6}{3 n-8}} \\
& -\left[\frac{4 n k \alpha \beta}{(n k+\gamma)^{2}}-\frac{\epsilon_{4} n^{2} k \delta}{4(n-2)}\right] \int_{\Omega}\left|\nabla V^{\frac{n k+\gamma}{2}}\right|^{2} d x
\end{aligned}
$$

Now, we can choose $\delta$ small enough to make the coefficient $\frac{4 n k \alpha \beta}{(n k+\gamma)^{2}}-\frac{\epsilon_{4} n^{2} k \delta}{4(n-2)}>0$. Hence, we have

$$
\varphi_{2}^{\prime}(t) \leq k_{5}+k_{6}^{\frac{3 n-6}{3 n-8}}
$$

where $k_{5}=\epsilon_{3} \int_{\Omega} a(x)^{\frac{1}{\epsilon_{3}}} d x$ and $k_{6}=\frac{\epsilon_{4} n k(3 n-8) C(\delta)}{4(n-2)}$.

Then, integrating (3.40) from 0 to $t$ yields that

$$
\int_{\varphi_{2}(0)}^{\varphi_{2}(t)} \frac{d \eta}{k_{5}+k_{6} \eta^{\frac{3 n-6}{3 n-8}}} \leq t
$$

If $u$ blows up in the measure $\varphi_{2}(t)$ as $t \rightarrow t^{*}$, then we can obtain the lower bound

$$
t^{*} \geq \int_{\varphi_{2}(0)}^{+\infty} \frac{d \eta}{k_{5}+k_{6} \eta^{\frac{3 n-6}{3 n-8}}} .
$$


Similar to the above derivation of (3.14) and (3.25), it is easy to get

$$
\varphi_{2}(t) \geq\left(\frac{4 k_{6}}{3 n-8}\right)^{-\frac{3 n-8}{2}}\left(t^{*}-t\right)^{-\frac{3 n-8}{2}} .
$$

This completes the proof of Theorem 5 .

\section{Acknowledgements}

The authors would like to express their thanks to the editor and the referees for their helpful comments. This work is supported by the NSF of China (11801108, 11701116), the Scientific Program (2016A030310262) of Guangdong Province, and the College Scientific Research Project (YG2020005) of Guangzhou University. Dr.Huafei Di also specially appreciates Prof.Yue Liu for his invitation of visiting to the University of Texas at Arlington.

\section{References}

[1] A. B. Al'shin and A. G. Sveshnikov M. O. Korpusov. Blow-up in nonlinear Sobolev type equations. De Gruyter Series in Nonlinear Analysis and Applications, 15, Walter de Gruyter \& Co., Berlin, 2011.

[2] D. G. Aronson. The porous medium equation. Nonlinear diffusion problems. Springer, Heidelberg, Berlin, 1986.

[3] D. G. Aronson and L. A. Peletier. Large time behaviour of solutions of the porous medium equation in bounded domains. J. Differ. Equations, 39(3):378-412, 1981.

[4] P. Biler and G. Karch C. Imbert. The nonlocal porous medium equation: Barenblatt profiles and other weak solutions. Arch. Ration. Mech. Anal., 215(2):497-529, 2015.

[5] V. A. Galaktionov et al. On unbounded solutions of the Cauchy problem for the parabolic equation $u_{t}=\nabla\left(u^{\sigma} \nabla u\right)+u^{\beta}$. Dokl. Akad. Nauk SSSR., 252(6):1362-1364, 1980 .

[6] V. A. Galaktionov and H. A. Levine. A general approach to critical Fujita exponents in nonlinear parabolic problems. Nonlinear Anal., 34(7):1005-1027, 1998.

[7] Y. Hu and X. C. Chen L. W. Wang. Lower bounds for blow-up time of porous medium equation with nonlinear flux on boundary. Int. J. Math. Anal. (Ruse), 7(53-56):26712676, 2013.

[8] Z. X. Jiang and X. F. Song S. N. Zheng. Blow-up analysis for a nonlinear diffusion equation with nonlinear boundary conditions. Appl. Math. Lett., 17(2):193-199, 2004.

[9] T. Kawanago. Existence and behaviour of solutions for $u_{t}=\Delta\left(u^{m}\right)+u^{l}$. Adv. Math. Sci. Appl., 7(1):367-400, 1997. 
[10] H. A. Levine. The role of critical exponents in blowup theorems. SIAM Rev., $32(2): 262-288,1990$.

[11] S. Z. Lian and C. C. Liu. On the existence and nonexistence of global solutions for the porous medium equation with strongly nonlinear sources in a cone. Arch. Math. (Basel), 94(3):245-253, 2010.

[12] G. M. Lieberman. Second order parabolic differential equations. World Scientific Publishing Co., Inc, River Edge, New Jersey, 1996.

[13] Y. Liu. Lower bounds for the blow-up time in a non-local reaction diffusion problem under nonlinear boundary conditions. Math. Comput. Modelling, 57(3-4):926-931, 2013.

[14] X. S. Lv and X. F. Song. Bounds of the blowup time in parabolic equations with weighted source under nonhomogeneous Neumann boundary condition. Math. Methods Appl. Sci., 37(7):1019-1028, 2014.

[15] L. Ma and Z. B. Fang. Blow-up analysis for a reaction-diffusion equation with weighted nonlocal inner absorptions under nonlinear boundary flux. Nonlinear Anal. Real World Appl., 32:338-354, 2016.

[16] L. E. Payne and S. Vernier Piro G. A. Philippin. Blow-up phenomena for a semilinear heat equation with nonlinear boundary condition, I. Z. Angew. Math. Phys., 61(6):999-1007, 2010.

[17] L. E. Payne and S. Vernier Piro G. A. Philippin. Blow-up phenomena for a semilinear heat equation with nonlinear boundary condition, II. Nonlinear Anal., 73(4):971-978, 2010 .

[18] L. E. Payne and P. W. Schaefer. Lower bounds for blow-up time in parabolic problems under Neumann conditions. Appl. Anal., 85(10):1301-1311, 2006.

[19] L. E. Payne and P. W. Schaefer. Lower bounds for blow-up time in parabolic problems under Dirichlet conditions. J. Math. Anal. Appl., 328(2):1196-1205, 2007.

[20] L. A. Peletier. Asymptotic behavior of solutions of the porous media equation. SIAM J. Appl. Math., 21:542-551, 1971.

[21] F. Quirós and J. L. Vázquez. Asymptotic behaviour of the porous media equation in an exterior domain. Annali della Scuola Normale Superiore di Pisa-Classe di Scienze, 28(2):183-227, 1998.

[22] J. Rubinstein and P. Sternberg. Nonlocal reaction-diffusion equations and nucleation. IMA J. Appl. Math., 48(3):249-264, 1992.

[23] J. C. Song. Lower bounds for the blow-up time in a non-local reaction-diffusion problem. Appl. Math. Lett., 24(5):793-796, 2011. 
[24] X. F. Song and X. S. Lv. Bounds for the blow-up time and blow-up rate estimates for a type of parabolic equations with weighted source. Appl. Math. Comput., 236:78-92, 2014 .

[25] G. S. Tang and X. T. Yang Y. F. Li. Lower bounds for the blow-up time of the nonlinear non-local reaction diffusion problems in $\mathbb{R}^{N}(N \geq 3)$. Bound. Value Probl., 265:1-5, 2014.

[26] J. L. Vázquez. The porous medium equation. Oxford Mathematical Monographs, Oxford University Press, Oxford, 2007. 